\title{
The Face of Clostriodes Difficile Infections in the Outpatient Setting
}

Michael S Wang, M.D., FACP, FIDSA ${ }^{1}$ @ , Samad Faheem, D.O. ', Joanna Mangio, D.O. ', Kevin Pham, D.O. ', Daniel Lloyd, D.O. ', Brianna Hatch-Vallier, D.O. ', Ewanah Johnson, M.D. ${ }^{1}$

${ }^{1}$ Medicine, Spectrum Health-Lakeland

Keywords: clostriodes difficile infection, ambulatory, severity markers

https://doi.org/10.51894/001c.12883

\section{Spartan Medical Research Journal}

Vol. 5, Issue 1, 2020

\begin{abstract}
BACKGROUND
It has long been well-established that Clostridiodes difficile infections (CDI) can cause severe morbidity and mortality. However, most of the literature to date has focused on hospital-diagnosed infections with less emphasis on clinic-based CDI cases. Guidelines from the 2010 IDSA/SHEA for CDI advocate for metronidazole as first-line therapy for mild to moderate CDI cases. However, the 2017 guidelines recommend oral vancomycin or fidaxomicin as first-line therapy due to their superior efficacy. Objective: The purpose of this study was to compare Clostriodes difficile infections in convenience samples of clinic vs. hospital patients.
\end{abstract}

\section{METHODS}

In 2019, a retrospective, case-controlled study was performed by the first six authors between 2015-2017 (i.e., prior to the 2017 IDSA/SHEA CDI guidelines) to compare ambulatory and hospital CDI treatment prescriptions. Analytic data included frequency of White blood cells (WBC) and creatinine collection, frequency of severe CDI cases, compliance with the 2010 guidelines, CDI recurrence, and mortality.

\section{RESULTS}

An eligible subgroup of $\mathrm{N}=92$ hospital patients at Spectrum Health Lakeland were more likely to have WBC (98.4\% vs $32.6 \%$, p<0.001) and creatinine (97.8 vs. 39.4, P < 0.001$)$ drawn than 184 patients receiving clinic-based care. Hospital sampled patients were more likely to have severe CDI $(46.7 \%$ vs $6.7 \%, \mathrm{p}<0.001)$. Mortality was less common in hospital patients $(1.1 \%$ vs. $7.6 \%, \mathrm{p}=0.017)$ and the recurrence rates were similar. $(21.2 \%$ inpatient vs. $28.3 \%$ outpatient, $(\mathrm{p}=0.224)$.

\section{CONCLUSIONS}

Based on these results, assessment of CDI severity remains limited in the ambulatory population due to the lack of severity markers. It is unclear if this is due to lack of available laboratory resources or difference in clinical presentation. Of those sample patients who have available markers of severity, patients receiving clinic-based diagnoses were less likely assessed to have severe CDI. Keywords: Cloistriodes difficile infection, ambulatory, severity markers

\section{INTRODUCTION}

Clostridiodes difficile (CDI) infections have produced significant morbidity and mortality in the United States. ${ }^{1,2}$ Although much of the CDI literature to date has been focused on hospital patients, there is increasing concern over the burden of CDI in ambulatory settings. ${ }^{1,2}$ Risk factors for community-acquired CDI are similar to hospital acquired CDI, including antibiotics, particularly high-risk antibiotics (e.g., clindamycin, fluoroquinolones, cephalosporins, and beta-lactams/lactase inhibitors). ${ }^{1}$

In a study of 14,453 patients across 10 sites, only $24 \%$ of cases were diagnosed in the hospital setting. ${ }^{2}$ In addition, patients thought to have community-acquired CDI have often had prior exposures in emergency rooms, dialysis centers, and physicians' offices ${ }^{1,2}$ Recurrent CDI is also common in both the health care (14\%) and community acquired settings (21\%). ${ }^{2}$

In terms of CDI treatment recommendations, the previously established treatments have included oral metronidazole $500 \mathrm{mg}$ every 8 hours and oral vancomycin $125 \mathrm{mg}$ four times per day. ${ }^{3}$ According to the 2010 Infectious Diseases Society/Society of Hospital Epidemiology of America (IDSA/SHEA) CDI guidelines, vancomycin was previously recommended for severe CDI over metronidazole, although metronidazole had been recommended for mild to moderate CDI. ${ }^{3,4}$ Oral fidaxomicin $200 \mathrm{mg}$ twice daily has also 
been shown to be efficacious for CDI. ${ }^{5}$

The most recent 2017 IDSA/SHEA guidelines now also recommend oral fidaxomicin or vancomycin for mild to moderate CDI cases unless the supply of the two medicines is limited or unavailable. ${ }^{4}$ The definition of severe CDI defined in both CDI guidelines has focused primarily on leukocyte count (> 15 cells $3 / \mathrm{uL}$, normal $4.5-11.0$ ) or creatinine > $1.5 \mathrm{x}$ baseline (normal creatinine $0.5-1.0 \mathrm{mg} / \mathrm{dL}$ ). ${ }^{4,6}$ Severity markers often influence CDI treatment options, specifically the decision of whether to use vancomycin, metronidazole, or fidaxomicin. 3,4,6 There may be limitations of these severity markers in certain populations, particularly those with hematologic malignancies. ${ }^{7}$ Our study sought to examine the utility of CDI markers in an ambulatory population versus an inpatient population.

It remains unclear what proportion of clinic-based physicians have the readily available laboratory assays when assessing patients for CDI. It is also unclear to what extent providers adhere to guidelines as recommended by the IDSA/SHEA. The purpose of this case-control study was to examine diagnostic and treatment patterns of outpatient CDI.

\section{METHODS}

This was a single-center, retrospective, case control study, comparing patients in an clinic-based patient settings vs. patients in a hospital setting. The $C$ Difficile Polymerase Chain Reaction (PCR) database was reviewed from the microbiology records, dating from January 2015 to September 2017. This study was conducted during the period from February 2019-August 2019 at Spectrum Health-Lakeland, a 200-bed community-based hospital with both teaching and non-teaching patient services in St. Joseph, MI.

The study hospital has a Level 3 Trauma Center with adult intensive care, medical, oncology, post-surgical, orthopedic, neurology, cardiac, and pediatric units. The Spectrum Health-Lakeland Institutional Review Board approved the study prior to data collection.

Case patients were defined as patients $\geqslant 18$ years with a positive $C$. difficile stool specimen collected in an ambulatory setting. Case patients were excluded from the analytic sample if there was no reported diarrheal illness (i.e., $\geqslant$ three watery stools in a 24-hour period) associated with the positive stool specimen or they could not be matched to a control within 90 days of the specimen collection date. ${ }^{1}$ Control patients were defined as CDI patients diagnosed in a hospital setting.

Emergency Department cases were defined as a hospital setting and included as part of the control group. Each case was matched to two controls by sex and age within 10 years and matched within six months of diagnosis of CDI.

Patients were also be excluded if they had a prior CDI diagnosis within the prior six months. Additional data measures included white blood cell count (WBC), creatinine, recent antibiotic use within the last 90 days, recent hospitalizations, Charlson Comorbidity Index (CCI), ${ }^{8}$ and treatment medications prescribed. The CCI is a method of measuring the prognostic impact of comorbid disease, which factors in disease processes such as heart disease, kidney disease, liver failure, diabetes, and malignancy. ${ }^{8}$

A healthcare associated CDI was defined as an infection in any patient who had an overnight stay in a health care facility (i.e., hospital or nursing home) during the prior 12 weeks. ${ }^{1}$ Other measured outcomes included mortality and recurrent CDI among surviving patients.

Statistical methods included the use of the chi-square test of association, Fisher's Exact Probability Test, and ANOVA. Computations were performed via the VassarStats computational software ${ }^{9}$ by the first author (MW).

\section{RESULTS}

During the study period, a total of 439 ambulatory patients with CDI (case) and 208 hospital patients with CDI (control) were initially identified. Of these patients, 255 case patients and 116 control patients were excluded based on age or CDI recurrence. A total of 92 (33.3\%) eligible case patients and 184 (66.7\%) control patients were identified.

The mean age in the case group was $61.7(\mathrm{SD}=17.9)$ and the mean age in the control group was $63.4(\mathrm{SD}=16.4), \mathrm{p}=$ 0.443. (Table 1) Case patients were less likely to have been on recent antibiotics than control patients (6.9\% vs. 46.7 , p $<0.001)$. Case patients were also less likely to have health care CDI than control patients $(22.8 \%$ vs. $53.8 \%$, p < 0.001$)$.

Case patients were also less likely to have had both WBC (32.6\% vs. 98.4\%, p < 0.001 ) and creatinine (28.3\% vs. $97.8 \%$, $\mathrm{p}<0.001)$ levels drawn. In addition, case patients were also less likely to have significant morbidity, based on composite CCI scores ${ }^{7}$ (1.8 [0-12] vs. 3.2 [0-12], P < 0.001). Although the mean WBC between the subgroups was not significantly different, case patients were less likely to have a WBC $>15$ cells $^{3} / \mathrm{uL}(6.7 \%$ vs. $21.5 \%, \mathrm{p}=0.040)$ and creatinine $1.5 \mathrm{x}$ baseline (3.8\% vs. $23.3 \%, \mathrm{p}=0.013)$.

The authors' ability to assess severity of CDI was diminished in case patients as compared to controls. (32.6\% vs. $98 \%, \mathrm{p}<0.001)$. Among patients in which CDI severity could be assessed (Table 2), cases were less likely to be have severe CDI $(6.7 \%$ vs. $46.7 \%, \mathrm{p}<0.001)$. Case patients were more likely to be treated with oral metronidazole (72.3\% vs $49.5 \%, \mathrm{p}=0.001$ ) and control patients were more likely to be treated with either oral vancomycin or fidaxomicin $(54.9 \%$ vs $25.8 \%, \mathrm{p}<0.001)$.

Thirty-day mortality occurrences were higher for control patients (7.6\% [14 of 184]) than case patients (1.1\% [1 of 92], $\mathrm{p}=0.017$ ). After accounting for mortality, recurrence of CDI within 60 days was similar in case $(28.3 \%)$ and control patients $(21.2 \%, \mathrm{p}=0.224)$.

\section{DISCUSSION}

To our knowledge, this is the first study to examine the assessment and treatment of severe CDI cases comparing only where patients were diagnosed (clinic patient vs. hospital patient). Previous studies involving outpatients have focused on location of exposure, specifically community-acquired vs. hospital-acquired CDI. ${ }^{1,10}$ Earlier studies have also found that there are clinical care exposures, including physician's offices, physical therapist, hemodialysis, and outpatient surgery centers, not previously classified as health care associated CDI. ${ }^{1}$

As physicians in an emergency room setting presumably 


\begin{tabular}{llll}
\hline & Ambulatory patients (Case) & Hospital patients (Control) & P value \\
Age & $61.7+17.9$ & $63.4+16.4$ & 0.443 \\
$\%$ Male & $32 / 92(34.8 \%)$ & $34.8 \%(64 / 184)$ & 1.000 \\
Nap1 ${ }^{+}$ & $12.0 \%$ & $11.4 \%$ & 0.520 \\
Health Care Associated & $22.8 \%(22 / 92)$ & $53.8 \%(99 / 184)$ & $<0.001$ \\
Charlson Comorbidity Index & $1.8+2.3$ & $3.2+2.4$ & $<0.001$ \\
Verified antibiotics within last 90 days & $46.7 \%(43 / 92)$ & $69.0 \%(127 / 184)$ & $<0.001$ \\
$\quad$ Fluoroquinolones & $14.1 \%(13 / 92)$ & $21.2 \%(39 / 184)$ & 0.192 \\
$\quad$ Penicillins & $8.7 \%(8 / 92)$ & $3.8 \%(7 / 184)$ & 0.156 \\
$\quad$ Beta-lactamase inhibitors & $7.6 \%(7 / 92)$ & $16.3 \%(30 / 184)$ & 0.060 \\
$\quad$ Cephalosporins & $14.1 \%(13 / 92)$ & $39.7 \%(73 / 184)$ & $<0.001$ \\
$\quad$ Marolides & $1.1 \%(1 / 92)$ & $3.8 \%(7 / 184)$ & 0.285 \\
$\quad$ Clindamycin & $1.1 \%(1 / 92)$ & $4.3 \%(8 / 184)$ & 0.280 \\
$\quad$ Trimethoprim & $2.2 \%(2 / 92)$ & $4.9 \%(9 / 184)$ & 0.346 \\
$\quad$ Carbapenem & $3.3 \%(3 / 92)$ & $3.3 \%(6 / 184)$ & 1.000 \\
WBC drawn & $28.3 \%(30 / 92)$ & $98.4 \%(181 / 184)$ & $<0.001$ \\
Creatinine drawn & $39.4 \%(26 / 92)$ & $97.8 \%(180 / 184)$ & $<0.001$ \\
\hline
\end{tabular}

+ North American pulsed-field gel electrophoresis type 1 strain

Table 2 - Clinical Data of Patients with CDI

\begin{tabular}{|c|c|c|c|}
\hline & $\begin{array}{l}\text { Ambulatory patients } \\
\text { (Case) }\end{array}$ & $\begin{array}{l}\text { Hospital patients } \\
\text { (Control) }\end{array}$ & $\begin{array}{l}P \\
\text { value }\end{array}$ \\
\hline WBC & $10.1+4.4$ & $11.7+6.6$ & 0.221 \\
\hline$\% W B C>15$ cells $^{3} / u L$ & $6.7 \%(2 / 30)$ & $21.5 \%(39 / 181)$ & $\begin{array}{l}< \\
0.040\end{array}$ \\
\hline Creatinine (mg/dL) & $1.0+0.6$ & $1.6+1.9$ & 0.125 \\
\hline Creatinine $>1.5 \times$ baseline & $3.8 \%(1 / 26)$ & $23.3 \%(42 / 180)$ & $\begin{array}{l}< \\
0.013\end{array}$ \\
\hline Albumin & $3.5+0.6$ & $3.4+0.7$ & 0.538 \\
\hline Ability to determine severity & $32.6 \%(30 / 92)$ & $98.0 \%(182 / 184)$ & $\begin{array}{l}< \\
0.001\end{array}$ \\
\hline Severe C DIFF & $6.7 \%(2 / 30)$ & $46.7 \%(85 / 182)$ & $\hat{0} .001$ \\
\hline Compliance with 2010 guidelines 6 & $53.8 \%(14 / 26)$ & $51.6 \%(94 / 182)$ & 0.501 \\
\hline$\%$ treated with metronidazole & $72.3 \%(48 / 66)$ & $49.5 \%(91 / 184)$ & $\begin{array}{l}< \\
0.001\end{array}$ \\
\hline$\%$ treated with oral vancomycin or fidaxomicin & $25.8 \%(17 / 66)$ & $54.9 \%(101 / 184)$ & $\hat{0} 0.001$ \\
\hline $\begin{array}{l}\% \text { non-compliance due to using vancomycin instead of } \\
\text { metronidazole (non-severe) }\end{array}$ & $83.3 \%(10 / 12)$ & $37.5 \%(33 / 88)$ & 0.004 \\
\hline Death within 30 days & $1.1 \%(1 / 92)$ & $7.6 \%(14 / 184)$ & 0.017 \\
\hline Recurrence within 60 days & $28.3 \%(26 / 92)$ & $21.2 \%(36 / 170)$ & 0.224 \\
\hline
\end{tabular}

have access to hospital resources, emergency room cases were classified as hospital patients. In addition, hospital patients were more likely to have significant co-morbidities, based on the difference in their $\mathrm{CCI}^{7}$ scores. $(\mathrm{p}<0.001)$ Not surprisingly, hospital patients were more likely to have health care-associated CDI than ambulatory patients, given their overall higher relative morbidity. $(p=0.017)$

In our sample, outpatients were less likely to have either WBC or creatinine levels drawn. As a result, our ability to assess disease severity comparisons in our hospital sample patients was limited $(\mathrm{p}<0.001)$, since CDI severity markers rely on such laboratory markers. ${ }^{3,4,6}$ This difference may 
be accounted for by either the differences in presentation or typical laboratory resources seen in outpatient settings. The higher severity of inpatients, may be due to the higher underlying morbidity within that population, as demonstrated by the difference in the CCI ( $<<0.001)$, although the comparison may be limited due to the relative lack of outpatient CDI data.

Although our ability to assess severe CDI was limited in case (ambulatory) patients, severe CDI was rare. Depending on the availability of vancomycin and fidaxomicin, this also raises issues as to the optimal potential treatment regimens for CDI. For those clinic-based providers who opt to use metronidazole, this raises issues due to possibility of missing potential cases of severe CDI and treatment appropriateness. $3,4,6,11-13$

In this sample, physicians in the control group (hospital patient) were more likely to adhere to the 2010 CDI guidelines. (Table 2) However, this difference was likely accounted for mostly by a significant number of clinic-based physicians prescribing oral vancomycin instead of metronidazole, which is now consistent with first-line therapy according to the 2017 IDSA Guidelines. ${ }^{4}$

Metronidazole was previously listed as first-line therapy for mild to moderate CDI infections in the 2010 guidelines, ${ }^{6}$ but has been discontinued as first therapy in the 2017 guidelines. ${ }^{4}$ Although outcomes between metronidazole and vancomycin for milder CDI cases in another study have been initially found to be similar, ${ }^{3}$ a subsequent study demonstrated inferior outcomes of symptom resolution and recurrence for metronidazole. ${ }^{11}$

However, there are still providers who opt to use metronidazole for milder cases of CDI due to the significant cost of vancomycin and fidaxomicin. 3,12,14 One recent study examined patients under 65 and found no significant differences between the use of vancomycin and metronidazole in milder cases. ${ }^{13}$ After factoring in patient mortality, our study demonstrated no differences mortality among surviving patients between outpatients and inpatients. ${ }^{3,6,11}$

This again raises the question of metronidazole prescriptions in the clinic setting, as these findings are not consistent with the 2017 CDI guidelines. ${ }^{4}$ As other modalities such as fecal transplants and bezlotoxomab, a monoclonal antibody, become increasingly become part of treatment regimens, ${ }^{15-17}$ the complexities of treating outpatients may only increase.
Strengths of this study include our case-control design, as we were able to generate a well-matched comparison group between hospital and ambulatory patients. In addition, our electronic health record (EHR) allowed us to obtain reliable data regarding concomitant results between stools and serum blood draws.

Although most providers at our institution used the same EHR, one study limitation was that there were a few providers who do not use the common EHR, perhaps influencing the availability of pharmaceutical data. In addition, many of the patients in the case group, specifically in the outpatient setting, did not have severity markers measured, but measuring the percentage of available markers was part of the study design.

\section{CONCLUSIONS}

As clinic-based cases of CDI has been shown to impose a high burden, 1,10,18 we have demonstrated some of the limitations in diagnosing and treating CDI in ambulatory patients. Future studies are required to examine diagnostic and treatment patterns and whether ambulatory providers adhere to updated CDI guidelines. Future studies could investigate the awareness of the new guidelines with respect to outpatient providers as compared to inpatient providers.

Based on these results, assessing CDI severity in the clinic settings remains challenging given the limited availability of laboratory diagnostics for clinic-based providers to check severity markers. Although our study demonstrated that severe CDI in outpatients is rare, there is still considerable debate iconcerning the role of metronidazole for CDI. Given the diagnostic challenges and frequent recurrences, clinic-based providers should exercise caution before prescribing metronidazole.

\section{CONFLICT OF INTEREST}

The authors declare no conflict of interest.

\section{FUNDING}

The authors report no external funding source for this study.

Submitted: February 15, 2020 EST, Accepted: May 15, 2020 EST

This is an open-access article distributed under the terms of the Creative Commons Attribution 4.0 International License (CCBY-4.0). View this license's legal deed at http://creativecommons.org/licenses/by/4.0 and legal code at http://creativecommons.org/licenses/by/4.0/legalcode for more information. 


\section{REFERENCES}

1. Guh AY, Adkins SH, Li Q, et al. Risk Factors for Community-Associated Clostridium difficile Infection in Adults: A Case-Control Study. Open Forum Infect Dis. 2017;4(4):1-8.

2. Lessa FV, Mu Y, Bamberg WM. Burden of Clostridium difficile in the United States. $N$ Engl J Med. 2015;372:825-834.

3. Zar FA, Bakkanagar SR, Moorthi KMLST, Davis MD. A Comparison of Vancomycin and Metronidazole for the Treatment of Clostridium difficile-Associated Diarrhea, Stratified by Disease Severity. Clin Infect Dis. 2007;45:302-307.

4. Mcdonald LC, Gerding DN, Johnson S. Clinical Practice Guidelines for Clostridium difficile Infection in Adults and Children: 2017 Update by the Infectious Diseases Society of America (IDSA) and Society for Healthcare Epidemiology of America (SHEA). Clin Infect Dis. 2018;66(7):e1-e48.

5. Thomas JL, Miller MA, Mullane KM. Fidaxomicin versus Vancomycin for Clostridium difficile Infection. N Engl J Med. 2011;364:422-431.

6. Cohen SH, Gerding DN, Johnson S, et al. Clinical Practice Guidelines for Clostridium difficile Infection in Adults: 2010 Update by the Society for Healthcare Epidemiology of America (SHEA) and the Infectious Diseases Society of America (IDSA). Infect Control Hosp Epidemiol. 2010;31(05):431-455. doi:10.1086/65 $\underline{1706}$

7. Wang MS, Evans CT, Rodriguez T. Clostridium difficile infection and limitations of markers for severity in patients with hematologic malignancy. Infect Control Hosp Epidemiol. 2013;34(2):127-132.

8. Charleson M, Pompei P, Ales K, MacKenzie C. A new method of classifying prognostic comorbidity in longitudinal studies: Development and validation. J Chronic Dis. 1987;40(5):373-383.

9. Lowry R. VassarStats: Website for Statistical Computation [Internet]. http://vassarstats.net/.

10. Delate T, Albrecht G, Won K, et al. Ambulatorytreated Clostridium difficile infection: A comparison of community-acquired vs. nosocomial infection. Epidemiol Infect. 2015;143:1225-1235.
11. Johnson S, Louie TJ, Gerding DN, et al Vancomycin, metronidazole, or tolevamer for Clostridium difficile infection: Results from two multinational, randomized, controlled trials. Clin Infect Dis. 2014;59(3):345-354.

12. Fabre V, Dzintars K, Avdic E. Role of Metronidazole in Mild Clostridium difficile Infections. Clin Infect Dis. 2018;67(12):1956-1958.

13. Appaneal HJ, Caffrey AR, LaPlante K. What Is the Role for Metronidazole in the Treatment of Clostridium difficile Infection? Results From a National Cohort Study of Veterans With Initial Mild Disease. Clin Infect Dis. 2019;69(8):1288-1295.

14. Gallagher JC, Reilly JP, Navalkele B, Downham G, Haynes K, Trivedi M. Clinical and Economic Benefits of Fidaxomicin Compared to Vancomycin for Clostridium Difficile Infection. Antimicrob Agents Chemother. 2015;59(11):7007-7010. doi:10.1128/aac.0 0939-15

15. Wilcox MH, Gerding DN, Poxton IR. Bezlotoxumab for Prevention of Recurrent Clostridium difficile Infection. $N$ Engl J Med. 2017;376(4):305-317.

16. Hocquart M, Lagier J-C, Cassir N, et al. Early Fecal Microbiota Transplantation Improves Survival in Severe Clostridium difficile Infections. Clinical Infectious Diseases. 2018;66(5):645-650. doi:10.1093/c $\underline{\mathrm{id} / \mathrm{cix} 762}$

17. Hota SS, Sales V, Tomlinson G, et al. Oral Vancomycin Followed by Fecal Transplantation Versus Tapering Oral Vancomycin Treatment for Recurrent Clostridium difficile Infection: An OpenLabel, Randomized Controlled Trial. Clinical Infectious Diseases. 2016;64(3):265-271. doi:10.1093/cid/ciw731

18. Kuntz JL, Johnson ES, Raebel MA, et al. Predicting the risk of Clostridium difficile infection following an outpatient visit: Development and external validation of a pragmatic, prognostic risk score. Clinical Microbiology and Infection. 2015;21(3):256-262. doi:1 $\underline{0.1016 / \text { i.cmi.2014.11.001 }}$ 